\title{
Elaboración de un portafolio electrónico para el desarrollo docente a través de una Unidad Didáctica Virtual Preparing an electronic portfolio for teaching development through a Virtual Teaching Unit
}

\author{
Susana Romero Yesa ${ }^{1}$, Marian Aláez ${ }^{2}$, Ana García-Olalla ${ }^{1}$, Mabel Segú ${ }^{1}$ \\ sromeroyesa@deusto.es, marian.alaez@deusto.es, ana.garciaolalla@deusto.es,msegu@deusto.es \\ ${ }^{1}$ Unidad de Innovación Docente \\ Universidad de Deusto \\ Bilbao, España \\ ${ }^{2}$ Facultad de Derecho \\ Universidad de Deusto \\ Bilbao, España
}

\begin{abstract}
Resumen- La docencia de calidad es una preocupación fundamental en las universidades. La evaluación acreditativa del profesorado a través de las agencias de calidad responde a esa inquietud. En la Universidad de Deusto, esta acreditación se estructura en tres niveles: la acreditación de la planificación de la docencia, la acreditación de su puesta en práctica y la revisión y mejora desde un enfoque de colegialidad. En esta comunicación nos centramos en la segunda. El profesorado se debe presentar a una evaluación acreditativa mediante la preparación de un portafolio docente. La Unidad de Innovación Docente ofrece talleres en los que los coordinadores de los centros acompañan a los profesores en su elaboración. Además de esta formación presencial, el profesorado necesita contar con documentación de apoyo accesible y actualizada. Para dar respuesta a esta necesidad, se ha elaborado en la plataforma institucional tipo Moodle, mediante un proyecto de innovación docente, una Unidad Didáctica Virtual (UDV) de apoyo a la formación presencial. En ella se ha elaborado una plantilla y se han incluido recursos como presentaciones, vídeos, foros y ejemplos de las evidencias a presentar. Los resultados han sido muy satisfactorios en relación con el cumplimiento de los objetivos establecidos e indicadores de impacto.
\end{abstract}

Palabras clave: Aprendizaje híbrido, Evaluación de la calidad docente, Formación de profesorado universitario

Abstract- Quality teaching is a fundamental concern in universities. The accreditation of teaching staff through quality assurance agencies responds to this commitment. At the University of Deusto, this accreditation is structured on three levels: the accreditation of teaching planning, the accreditation of its implementation and the accreditation in the review and improvement from a collegiality approach. In this paper we focus on the second one. The teaching staff must go through an accreditation assessment by preparing a teaching portfolio. The Teaching Innovation Unit offers workshops in which school coordinators accompany teachers in the preparation of their portfolios. In addition to this face-to-face training, teachers need accessible and up-to-date support documentation. In response to this need, through a teaching innovation project, a Didactic Virtual Unit (DVU) has been developed on the institutional Moodle platform to support face-to-face training. A template has been created and a variety of resources have been included, such as presentations, videos, forums and examples of each of the pieces of evidence to be presented. The results have been very satisfactory in relation to the fulfilment of the established objectives and impact indicators.
Keywords: B-Learning, Evaluation of teaching quality, Training of university teaching staff

\section{INTRODUCCIÓN}

La mejora de la docencia pasa necesariamente por la revisión de la puesta en práctica de la misma. El proceso de creación y entrada en el Espacio Europeo de Educación Superior (EEES), ha traído consigo que muchos de los esfuerzos dirigidos a la mejora de la calidad de la docencia realizados tanto por los responsables de organismos gubernamentales, por las agencias de calidad e instituciones, como por el propio profesorado se hayan centrado en la planificación de las enseñanzas. Por parte de los primeros, estableciendo las directrices para que las enseñanzas se planifiquen y desarrollen cumpliendo unos ciertos criterios de calidad comunes, por parte de las instituciones mediante la elaboración de los planes de estudio de las nuevas titulaciones de grado y máster siguiendo esas directrices, y por parte del profesorado concretando esa planificación en los programas y/o guías docentes de aquellas enseñanzas que van a trabajar con su alumnado. Sobre este esquema de responsabilidad compartida se ha articulado un sistema de garantía de calidad de estas enseñanzas, en principio coherente sobre aquello que está escrito y previsto para su posterior desarrollo.

Sin embargo, somos también conscientes de que el verdadero reto de la calidad de la formación está en su puesta en práctica. Por lo que, transcurrida más de una década de la aplicación de dichos mecanismos de planificación, las preguntas clave deben centrarse ahora en qué y cómo se está aplicando lo planificado, $\mathrm{y}$ en qué medida todo ello ha servido para transformar los procesos de enseñanza-aprendizaje y mejorar los resultados de los mismos.

Sabemos que, en cumplimiento de las directrices de la ENQA (2005, 2015) los mecanismos establecidos para el seguimiento y acreditación de los títulos fueron diseñados con este objetivo, pero también sabemos por la experiencia en este tiempo de aplicación que se centran en cuestiones 
fundamentalmente generales y cuantitativas, que rara vez penetran en lo que realmente acontece en los procesos de enseñanza-aprendizaje que desarrolla el profesorado y estudiantado, de carácter eminentemente cualitativo, más allá de unos resultados de aprendizaje expresados en tasas.

Precisamente el Programa Docentia promovido por Aneca (Aneca, 2006, 2015), e impulsado en colaboración con las agencias autonómicas de calidad para su desarrollo, tiene como objeto la evaluación y promoción de la calidad de la docencia. A pesar de que entre sus objetivos se menciona apoyar a las universidades para que desarrollen esta calidad promoviendo el desarrollo profesional de su profesorado, la experiencia de una década de diseño y desarrollo del modelo nos indica que se ha centrado más en la evaluación de la docencia y menos en el desarrollo profesional, y que se ha realizado mediante procedimientos fundamentalmente cuantitativos, mimetizando procesos y procedimientos aprendidos para la calidad de los títulos.

Aunque este Programa Docentia se definiera como un mecanismo de garantía interna de las universidades, por entender que externamente es difícil y poco operativo promover el desarrollo profesional, lo cierto es que en pocos casos el programa ha servido para que las universidades definan un modelo propio de desarrollo profesional y que ello haya tenido un impacto claro y directo en la transformación de los modelos de formación y aprendizaje. Conscientes en parte de estas limitaciones, asistimos a la presentación por Aneca de una nueva versión revisada del Programa Docentia (Aneca, 2021) que en su declaración de intenciones plantea avanzar en esta dirección.

Por todo ello, se mantienen y cobran aún mayor relevancia estas preguntas clave sobre si se aplica la planificación elaborada, si ello sirve para transformar los procesos de enseñanza-aprendizaje y mejorar sus resultados, o bien, en caso de no ser así, qué y cómo se hace para mejorarlo. El trabajo que aquí se presenta trata de responder a estas preguntas planteando una experiencia desarrollada para recoger y compartir la práctica docente del profesorado mediante el empleo del portafolio docente en soporte digital, y cómo se puede acompañar al profesorado para facilitar su elaboración y para reflexionar sistemáticamente sobre su práctica a fin de mejorarla.

\section{CONTEXTO}

En el contexto anteriormente descrito, se desarrolla la experiencia que presentamos, realizada en la Universidad de Deusto, como ejemplo de un desarrollo del Programa Docentia con una orientación diferente a la mayoritariamente descrita en el estado de la cuestión, por estar orientada hacia un modelo de formación y modelo de desarrollo profesional previamente definidos (Deusto, 2007). Así, los mecanismos desarrollados internamente para evaluar la calidad de su docencia se orientan hacia dichos modelos, evaluando cómo el profesorado desarrolla las descripciones de buena práctica contenidas en los mismos (Villa y García-Olalla, 2014).

En primer lugar, la Universidad de Deusto, asumió la necesidad de una transformación en su sistema docente a nivel institucional, publicando en 2001 el diseño de su Marco
Pedagógico, su Modelo de Formación (MFUD) y su Modelo de Aprendizaje autónomo y significativo (MAUD) que contempla cinco fases: la contextualización basada en la experiencia, la observación reflexiva, la conceptualización, la experimentación activa y la evaluación (Deusto, 2001). El modelo de competencias en el que las universidades iban a basar sus titulaciones pasaba necesariamente porque el profesorado diseñara sus asignaturas orientándolas hacia el horizonte del perfil académico profesional de la titulación, utilizando para ello el eje de las competencias a desarrollar, y haciendo girar alrededor del mismo las estrategias de enseñanza-aprendizaje, actividades, contenidos y sistemas de evaluación. Era este nuevo modelo hacia el que había que orientar el proceso de innovación y el desarrollo profesional de sus docentes.

En coherencia con ese horizonte, para realizar la evaluación acreditativa de la docencia se han desarrollado tres procedimientos de evaluación cualitativa basados en evidencias: el Label 1, que evalúa la calidad de la planificación; el Label 2, que evalúa la calidad de la puesta en práctica de la docencia; y el Label 3, que evalúa su revisión y mejora desde un enfoque de colegialidad.

En el caso del Label 1, la recogida y análisis de las evidencias, resultó inicialmente más sencilla dada la existencia de dos instrumentos definidos que recogen dicha planificación: el programa y la guía de aprendizaje; cuya calidad se revisa mediante 3 criterios $y$ diez indicadores previamente establecidos.

Sin embargo, la cantidad de documentos, instrumentos, producciones e interacciones que se desarrollan en los procesos de enseñanza-aprendizaje en la interacción entre el profesorado y el alumnado es tan amplia y variada que, a priori, parecía sumamente difícil encontrar y acordar un conjunto de evidencias relevantes pero mensurables, recogerlas en algún tipo de soporte o soportes, y definir criterios acordados de calidad de su buena práctica. Con ese fin un equipo experimental de docentes se dedicó durante dos años a trabajar en un proyecto de Innovación (2011/12 y 2012/13). El resultado de su trabajo fue valorado con un alto grado de satisfacción por el equipo participante y dio como resultado un procedimiento, soporte, y documentación que puso a disposición de toda la comunidad educativa para difundir y extender institucionalmente ese proceso de acreditación del Label 2 (García-Olalla, 2014).

Durante aquella primera experiencia piloto (entre 2011 y 2013) se empezó por definir que la Acreditación del Label 2 tiene por objetivo asegurar la orientación y promoción del aprendizaje de los estudiantes hacia el logro de un aprendizaje autónomo y significativo, mediante una gestión adecuada de los métodos de enseñanza, el empleo del tiempo, los recursos materiales y personales, y el seguimiento y evaluación del proceso, a partir de la construcción de un clima y un compromiso con la exigencia, la profundidad y la calidad del trabajo y el aprendizaje.

Sin haber definido aún los criterios e indicadores mediante los que se demostraría esa buena práctica docente, se decidió que el portafolio docente sería el instrumento óptimo que permitiría poder recoger y ordenar evidencias que podían llegar a ser de naturaleza tan variada. Como expresara Fernández- 
March (2006:18), el portafolio supone "una descripción de los esfuerzos y resultados de un profesor por mejorar su enseñanza, incluyendo documentos y materiales que en conjunto muestran el alcance y la calidad del rendimiento docente del profesor. $\mathrm{Al}$ mismo tiempo que operan también dentro de los esfuerzos por mejorar los centros educativos y la enseñanza en cuanto profesión". Se trata de una historia documental estructurada de un conjunto de materiales seleccionados por el/la docente para mostrar el aprendizaje, reflexionando sobre él y sobre su evolución. Para ello el portafolio docente no debe ser una recopilación exhaustiva de los documentos y materiales que afectan a la actuación docente, sino presentar una información seleccionada sobre las actividades de enseñanza y una sólida evidencia de su efectividad. La dilatada experiencia desarrollada por Seldin (1997) y sus colaboradores trabajando en la elaboración de portafolios docentes había permitido mostrar y documentar sus beneficiosos efectos y resultados, tanto con finalidades formativas como sumativas.

El trabajo detallado sobre el tipo de evidencias a incluir y cómo ordenarlas, dio como resultado la formulación de 3 criterios que se concretan en 10 indicadores, en torno a los cuales se estructura el contenido del portafolio, y se realiza también su posterior revisión: gestión del aprendizaje (metodología, gestión del tiempo y recursos), orientación y tutoría (orientación y sistema de tutoría), y evaluación del aprendizaje (información, técnicas, retroalimentación y resultados de aprendizaje).

Pero, además, se extrajeron dos lecciones aprendidas de gran relevancia que, aunque no se hallaban entre los objetivos previstos de aquel proyecto, se han mantenido hasta la actualidad por el potencial transformador que revelaron desde su inicio:

- La realización del portafolio docente con un soporte digital facilita enormemente el archivo y la actualización de las evidencias documentales, y sus enlaces. Además, permite compartir en abierto los portafolios de otros docentes, y tener así acceso a numerosos ejemplos de estrategias para la puesta en práctica de la docencia.

- El profesorado participante en la experiencia valoró muy positivamente la dinámica de trabajo colaborativo surgida en el equipo de trabajo y la riqueza del intercambio de experiencias de la práctica docente que había permitido; realmente se habían sentido trabajando en una comunidad de práctica profesional.

Desde entonces la formación para la elaboración del portafolio docente orientada a la acreditación se organiza en forma de talleres en los que los coordinadores Label 2 de los centros acompañan a los docentes en este proceso. Por otra parte, dado que los docentes están acostumbrados en sus asignaturas al uso de la plataforma institucional, ALUD (basada en Moodle), se ha decidido que sea en una plataforma paralela a esta, DOCENTIA, donde se alojen los portafolios.

Por otra parte, la obtención del Label 1 se ha ido consolidando a lo largo de estos últimos años en la universidad y muchos de los profesores cuentan ya con esta acreditación. Sin embargo, el proceso de obtención del Label 2 aún no ha llegado a este nivel de consolidación, dado el mayor nivel de complejidad y compromiso que entraña. El procedimiento ha sido aplicado durante cinco cursos académicos (entre 20014/15 y 2018/19), hasta el curso 2019/20 en el que se da un nuevo paso que constituye la finalidad del proyecto de innovación que aquí presentamos.

Así, el Objetivo General de este proyecto de innovación docente es ayudar al profesorado de la Universidad de Deusto a elaborar un portafolio docente que permita la reflexión sobre la puesta en práctica de su docencia y la acreditación Label 2.

Se concretaron también los siguientes objetivos específicos:

- Implementar un curso para facilitar al profesorado la elaboración de portafolios docentes, y hacerlo siguiendo el ciclo de enseñanza-aprendizaje establecido en la Universidad (MAUD), dando así un ejemplo coherente al profesorado que debe hacer lo mismo en sus asignaturas (Gairín, 2011).

- Presentar de modo estructurado, actualizado y accesible documentación clave para el apoyo al taller presencial.

- Facilitar el acceso del profesorado de la UD, a material actualizado para la elaboración de portafolios, y a la estructura a seguir a través de una plantilla.

- Presentar ejemplos de actividades de cada una de las fases del MAUD.

- Poner a disposición del profesorado la experiencia acumulada en la evaluación externa de la puesta en práctica de la docencia Label 2 a través de un listado explicativo de los errores más frecuentes.

- Hacer partícipe a la Comunidad Universitaria del diseño final del curso, a través de la recogida, valoración e incorporación de sus sugerencias de mejora.

Y para lograr estos objetivos se propuso la creación de un curso para la elaboración de un portafolio docente, utilizando DOCENTIA como soporte, DRIVE como repositorio y formato Google para toda la documentación.

La plataforma DOCENTIA es un buen soporte para dicho apoyo, concretamente por la posibilidad de recoger la documentación de una forma estructurada, con comentarios explicativos de los contenidos y utilidad de los documentos ofrecidos; por permitir contar con los documentos a través de enlaces a carpetas Drive que garantiza su actualización; por la disponibilidad de un foro para preguntas-mensajes; y por la disponibilidad de tareas para realizar dentro y fuera del aula con programación de tiempos de entrega y sistema de ofrecimiento de feedback.

Además, la ubicación de este curso en la plataforma DOCENTIA, permitía un doble empleo: en los talleres presenciales como apoyo, y para el acceso autónomo del profesorado que así lo requiriera.

Las integrantes del grupo de profesoras que lo iba a llevar a cabo tenían experiencia en formación de profesorado en general y en el Label 2 en particular, y procedían de diferentes facultades, centros y campus, lo que auguraba un buen ajuste a las necesidades formativas del profesorado asistente a los cursos. Todo ello en un contexto en el que se pretendía mejorar 
los programas de formación (Escobar, 2015), diseñando planes $\mathrm{y}$ acciones formativas coherentes con las necesidades tanto del profesorado como de la propia institución (Fernández-March, 2003) y que integren también el desarrollo de su competencia digital (González y Raposo, 2008; Díaz y López, 2009; Romero, Castejón, López y Fraile, 2017).

\section{DESCRIPCIÓN}

El proyecto se basa en el uso de dos espacios virtuales, uno para la elaboración de una Unidad Didáctica Virtual (UDV) que pueda servir al profesorado para la realización de su portafolio, y los propios portafolios de los docentes, creados a partir de una plantilla y puestos a disposición del resto de la comunidad educativa.

En el caso de la UDV, se desarrolló en siete fases, con sus correspondientes actividades, recursos y tiempos:

- $1^{\text {a }}$ fase, apertura del curso en la plataforma institucional (septiembre - octubre, 2019): creación de la UDV; identificación de apartados; y construcción del esquema de contenidos.

- $2^{\mathrm{a}}$ fase, adaptación de contenidos previos (octubre diciembre, 2019): recopilación de materiales; adaptación al formato Google Drive; y ubicación dentro de la UDV.

- $3^{\mathrm{a}}$ fase, elaboración de nuevos contenidos (enero - mayo, 2020): identificación de nuevos contenidos; elaboración de los mismos teniendo en cuenta el recurso más apropiado (documentos, vídeos, presentaciones, tareas, cuestionarios, foro...), aplicando el formato Google Drive y las facilidades de la propia plataforma; ubicación dentro de la UDV; y redacción de explicaciones para el uso adecuado de cada uno de ellos.

- $4^{\mathrm{a}}$ fase, experimentación con un grupo de profesores y profesoras (junio - noviembre, 2020): utilización de la UDV en los talleres para la elaboración del portafolio correspondientes a la convocatoria 2020-2021.

- $5^{\mathrm{a}}$ fase, evaluación de la Unidad por distintos agentes para la recogida de sugerencias de mejora (junio, 2021): por los docentes que lo utilizan para la elaboración de su portafolio, por los coordinadores/as que lo utilizan de apoyo para la realización de talleres y por las personas encargadas de los departamentos.

- $6^{\text {a }}$ fase, evaluación de idoneidad de las sugerencias presentadas e incorporación a la UDV (junio - julio, 2021).

- $7^{\mathrm{a}}$ fase, divulgación (septiembre - octubre 2021): socialización de la existencia y uso de la UDV en la Comunidad Universitaria con las mejoras introducidas, así como los resultados del proyecto en un Congreso de Innovación Docente.

El otro espacio virtual creado en el proyecto es la plantilla (Figura 1) elaborada para facilitar la realización del portafolio al profesorado. Así, cuando un docente decide hacer su portafolio Label 2, realiza una petición al departamento de recursos TIC para que lo creen en la plataforma institucional en uno de los tres idiomas oficiales: castellano, euskera o inglés. La plantilla no es más que un "esqueleto de etiquetas" que sirven al docente para la organización de las evidencias que tiene que presentar, así como para no olvidarse de ninguna, pero es un elemento clave como ya se verá en los apartados de resultados/conclusiones. En la misma plantilla el docente dispone además del protocolo donde se explica el porqué de cada evidencia, la forma de presentarla, etc.

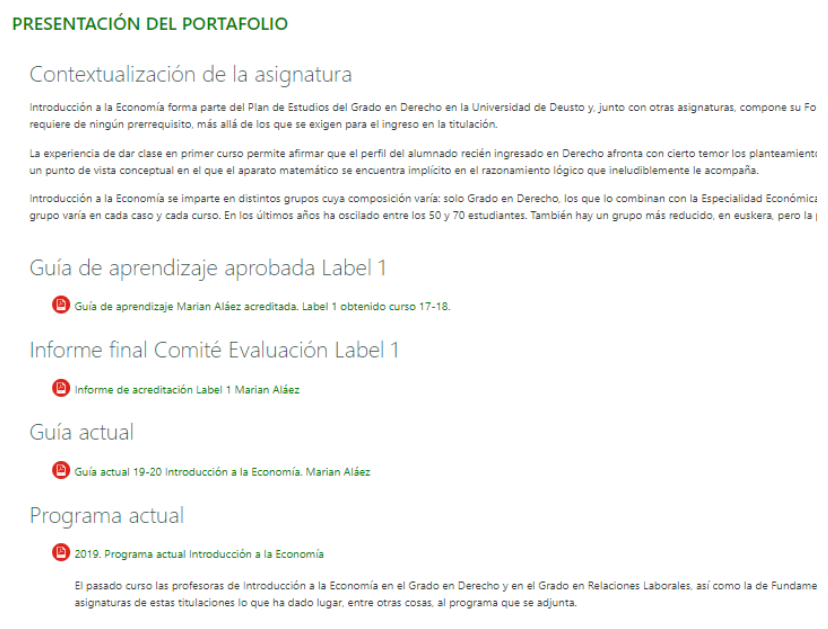

Figura 1. Primeros ítems de la plantilla creada ya rellena.

\section{Resultados}

Para conocer el impacto de este proyecto se identificaron los siguientes indicadores:

- INDICADOR 1: Elaborar la unidad didáctica en la fecha prevista y siguiendo el ciclo MAUD. La unidad, según el calendario inicial del proyecto, debiera haberse terminado en abril 2020. Sin embargo, con la declaración del estado de alarma y los cambios académicos que vinieron después, no se terminó hasta mayo 2020, una vez finalizadas las clases del curso académico.

- INDICADOR 2: Someter la unidad elaborada a la revisión por parte de participantes, responsables de departamento y coordinadores Label 2, con el fin de recabar sus sugerencias de mejora. Por la misma razón aducida en el indicador anterior, la unidad didáctica se presentó al mismo tiempo a todos los grupos de interés y no por fases, como estaba previsto. El envío del cuestionario ha tenido lugar en el segundo semestre de este curso 20-21 por considerar que los destinatarios tenían mayor conocimiento de causa para responder.

- INDICADOR 3: Obtener un mínimo de 3,5/5 en los ítems referidos a la disponibilidad de la documentación. Para medirlo, se han incorporado dos ítems en una encuesta de satisfacción: su percepción de satisfacción con el curso como ayuda a la preparación del portafolio y con la pertinencia de los contenidos y recursos de este. El resultado promedio ha sido de un $4,6 / 5$ en los dos indicadores.

- INDICADOR 4: Obtener un mínimo del 60\% de matriculaciones en el curso implementado de entre el profesorado que presenta portafolios en la convocatoria de Label 2 del curso 2020-2021. Aunque este indicador quedaba en un principio fuera del periodo de alcance del 
proyecto, se consideraba que era un indicador de importancia para conocer su acierto. En este caso, no disponemos de datos fiables dado el retraso que tuvo lugar en poner el curso a disposición de los docentes por los motivos anteriormente señalados. No obstante, se ha observado que los portafolios presentados a acreditación seguían la plantilla ofrecida junto con el curso.

- INDICADOR 5: Obtener un mínimo del 80\% de acreditaciones entre los profesores participantes de la Unidad Didáctica que se presentan en la convocatoria de Label 2 (2020-2021). Un 87\% del profesorado que se ha presentado a la convocatoria ha obtenido una acreditación total; el resto ha obtenido una acreditación condicionada, lo que significa que podrán obtenerla en la siguiente convocatoria. No ha habido "no acreditaciones". Sin embargo, estos son datos globales, ya que, como se ha comentado con anterioridad, no se dispone de datos fiables de quiénes han sido usuarios del curso elaborado y quiénes no.

- INDICADOR 6: Presentar la buena práctica en un congreso de Innovación Docente. Con la presentación de esta buena práctica quedaría cumplido este indicador.

Como se ha explicado, la situación de pandemia y el consiguiente foco del esfuerzo universitario en la adaptación de la docencia ha generado algunos retrasos que han afectado al resultado de los indicadores en cuanto a su plazo de cumplimiento. Por todo ello y debido a que se trataba de una situación de fuerza mayor, se pueden dar por cumplidos todos los indicadores con la excepción del Indicador 4, para el que se carece de datos. Asimismo, el Indicador 5 se ha tenido que transformar, también por el referido problema de datos. En cualquier caso, dado el convencimiento de la utilidad del trabajo elaborado a tenor de los comentarios recibidos en la encuesta, en la siguiente edición de evaluación acreditativa del profesorado, se recabarán los datos faltantes. Por todo lo señalado, entendemos que cabe afirmar que se ha logrado el impacto deseado.

El objetivo general propuesto "ayudar al profesorado de la Universidad de Deusto a elaborar un portafolio docente que permita la reflexión sobre la puesta en práctica de su docencia y la acreditación Label 2" se ha cubierto a través del cumplimiento de sus objetivos específicos:

- En la elaboración del curso, se ha seguido el ciclo del Modelo de Aprendizaje de la Universidad de Deusto (MAUD), reforzando la interiorización del modelo por parte del profesorado.

- Se ha facilitado el acceso del profesorado de Deusto, a material actualizado para la elaboración de portafolios. Históricamente la documentación necesaria, tanto los documentos oficiales como otros de carácter práctico para facilitar la elaboración de portafolios de las asignaturas era preparado por la Unidad de Innovación Docente y puesto a disposición de los profesores a través de los coordinadores de los centros vía mail. Mediante la creación del curso DOCENTIA en este proyecto se ha conseguido contar con un repositorio centralizado de todo el material necesario. Adicionalmente, al enlazar los documentos susceptibles de cambios con una carpeta de documentación en Drive, se ha garantizado su permanente actualización.
- Se ha presentado de modo estructurado y accesible documentación clave para la elaboración de portafolios. Dicha documentación se está utilizando por la coordinación de los centros como apoyo a los talleres que realizan con el profesorado y, de forma autónoma, algunos docentes. Esto ha sido posible gracias a la utilización de DOCENTIA como entorno de aprendizaje, ya que además de ficheros permite incorporar otro tipo de materiales como vídeos, enlaces, tareas, etc. todo ello de un modo ordenado y acompañado de comentarios explicativos.

- Por tratarse el portafolio de un instrumento para la recogida de evidencias y pedirse estas estructuradas en base al ciclo MAUD, se han incorporado ejemplos de actividades de cada una de las fases. Adicionalmente, se han compartido ejemplos de portafolios completos que han obtenido muy buen feedback por parte de los evaluadores. Estos últimos han señalado la buena evolución de los portafolios presentados, especialmente por una mejor estructuración de las evidencias, facilitada por la disponibilidad de la plantilla preparada al efecto.

- Se ha puesto a disposición del profesorado la experiencia acumulada en la evaluación externa de la puesta en práctica de la docencia Label 2 a través de un listado explicativo de los errores más frecuentes. Por tratarse de un enlace a un documento drive, su contenido podrá actualizarse de forma eficiente en cada edición.

- Se ha hecho partícipe a la Comunidad Universitaria del diseño final del curso, a través de la recogida, valoración e incorporación de sus sugerencias de mejora.

- Se ha difundido el curso a través de un Congreso.

\section{CONCLUSIONES}

Este proyecto ha ido encaminado, por una parte, a mejorar la calidad docente, a través de la puesta a disposición del profesorado de evidencias de buenas prácticas de docencia realizadas por sus compañeros. Por otra parte, se ha utilizado también para mostrar, al profesorado usuario, un ejemplo de docencia on-line que sigue las fases del MAUD. Este último aspecto cobra especial relevancia en un contexto de cambio apresurado de docencia presencial a modalidad en remoto.

La sostenibilidad del proyecto queda asegurada por la combinación necesidad de acreditación-utilidad de la unidad docente para lograrlo, constatado en las encuestas, además de por el hecho de no requerir nuevos aportes de recursos de ningún tipo, con la excepción de los dedicados a su actualización. Este último hito será llevado a cabo, anualmente, por la Unidad de Innovación Docente, responsable del programa DOCENTIA. Por otra parte, el hecho de que los coordinadores Label 2 lo utilicen en sus talleres anuales dirigidos al profesorado, asegura su continuidad.

Tras la experiencia lograda después de haber llevado a cabo unos años antes un proyecto análogo al aquí expuesto, orientado a la mejora de la planificación docente, cabe constatar su transferibilidad. La utilización de una plataforma tipo Moodle como apoyo al profesorado para la mejora de la calidad docente es trasladable a otros ámbitos, con las adaptaciones pertinentes. 
De hecho, tras la realización de ambos proyectos y su posterior evaluación, el equipo de docentes está valorando la posibilidad de transferir este proyecto al contexto de la obtención de la acreditación de la planificación docente en el ámbito de los postgrados, así como para recoger y poner a disposición del profesorado buenas prácticas de cada una de las fases del ciclo MAUD.

En el referido contexto de pandemia en el que ha tenido lugar esta práctica, merece resaltar la importancia del ingrediente tecnológico que incorpora. A través de su uso para solventar una clara necesidad de acreditación, el personal docente accede a un ejemplo de utilización de tecnologías y recursos digitales diversos que, sin duda alguna, transferirá a su propia docencia. Consideramos que el acompañamiento que han recibido en su utilización, tanto en la formación presencial como en la modalidad on-line ha sido fundamental para hacer posible esta transferencia.

Tras la evaluación del proyecto, se recomienda la utilización combinada de recursos variados, así como que algún equipo asuma el compromiso de atender de forma continuada las necesidades que puedan surgir en el profesorado en el uso de la plataforma. En el caso descrito en esta buena práctica, la asunción de esta responsabilidad está asegurada en tanto y cuanto está encomendada a la Unidad de Innovación Docente, compuesto por personal de distintos centros, con formación, motivación y asignación de tiempos para este cometido. Caso de no poder contar con este importante recurso, la práctica presentada no tendría garantías de ser efectiva.

\section{AgRADECIMIENTOS}

El proyecto descrito en esta comunicación no hubiera sido posible sin el apoyo proporcionado por la Universidad de Deusto a través de la XI Convocatoria de Proyectos de Innovación Docente.

\section{REFERENCIAS}

ANECA (2015). Programa DOCENTIA (Programa de apoyo para la evaluación de la actividad docente del profesorado universitario). Integración y actualización del programa 2006.v1. Recuperado

de http://www.aneca.es/content/download/13305/164819/fil e/DOCENTIA_nuevadoc_v1_final.pdf

ANECA (2021). Programa de apoyo para la evaluación de la calidad de la actividad docente del profesorado universitario. Última actualización 20/05/2021. Recuperado http://www.aneca.es/content/download/16186/197428/fil e/programaDOCENTIA_210527.pdf

Díaz, V. M., y López, M. A. R. (2009). La formación docente universitaria a través de las TICs. Pixel-Bit. Revista de Medios y Educación, (35), 97-103.
ENQA (2005). Standards and guidelines for Quality Assurance in European Higher Education Area. Helsinki: ENQA. Recuperado de https://enqa.eu/wpcontent/uploads/2015/09/ESG_3edition.pdf

ENQA (2015). Standards and Guidelines for Quality Assurance in the European Higher Education Area (ESG). Brussels: EURASHE. Recuperado de https://enqa.eu/wpcontent/uploads/2015/11/ESG_2015.pdf

Escobar, M. L. R. (2015). La formación docente de los profesores de nivel superior. ANFEI Digital, (1). Recuperado http://anfei.org.mx/revista/index.php/revista/article/view/ 2

Fernández-March, A. (2003). Formación pedagógica y desarrollo profesional de los profesores de universidad: análisis de las diferentes estrategias. Revista de educación, 331, 171-197.

Fernández-March, A. (2006). El portafolio docente como estrategia formativa y desarrollo profesional. Universidad de Deusto: Taller de Formación. Documento policopiado inédito.

Gairín, J. (2011). Formación de profesores basada en competencias. Bordón. Revista de Pedagogía, 63(1), 93108.

García-Olalla, A. (2014). El portafolio docente: un instrumento para evaluación y mejora de la práctica docente. Revista CIDUI, 2, 1-13. Recuperado de https://www.cidui.org/revistacidui/index.php/cidui/articl e/view/550

González, M., y Raposo, M. (2008). Necesidades formativas del profesorado universitario en el contexto de la convergencia europea. Revista de Investigación Educativa, 26(2).

Romero, M. R., Castejón, F. J., López, V. M., y Fraile, A. (2017). Evaluación formativa, competencias comunicativas y TIC en la formación del profesorado. Comunicar 25(52), 73-82.

SELDIN, P. (1997). The Teaching Portafolio. Bolton, MA: Anker Publishing Company Inc.

Universidad de Deusto (2001). Marco Pedagógico de la Universidad de Deusto. Bilbao: Universidad de Deusto.

Universidad de Deusto (2007). Manual para la garantía interna de calidad docente: modelo de desarrollo profesional en la UD. Bilbao: Universidad de Deusto.

Villa, A. y García-Olalla, A. (2014). Un sistema de garantía de calidad de la docencia: un estudio de caso. Revista Electrónica Interuniversitaria de Formación del Profesorado, 17(3), 65-78. doi: 10.6018/reifop.17.3.204061 